\title{
An Improved Hybrid Optimization Algorithm based on Iteration for Solving Aeroengine Model
}

\author{
D.W. Yin, R.Y. Lv, B. Chang \\ Naval Academy of Armament \\ Shanghai, China
}

\begin{abstract}
The solution Algorithm of the aeroengine nonlinear model is a classic problem, which is relatively mature in theory. But this question is still studied today, because the mathematical character of the aeroengine nonlinear model is so complex, that it is difficult to be convergent using the traditional iteration algorithm. So an idea is proposed to solve this problem, which is conversing the model solution to an optimization problem, and using optimization algorithms to solve the difficulties. In order to ensure the calculation speed, the iteration optimization method is chosen, and a new improved hybrid iteration optimization methods is design. In order to verify the improved algorithm, the simulation experiment was carried out taking an given turbofan engine as an example. The simulation results shows that, the solution precision is improved in some degree, when the new algorithm is used.
\end{abstract}

Keywords-Aeroengine; nonlinear equations; iteration; optimization algorithm

\section{INTRODUCTION}

Aeroengine component level analysis model and its solution method is very important, which is the base of many technologies, such as aeroengine performance analysis, control system design, digital simulation, and so on[1-3]. The aeroengine non-linear model can be describe as nonlinear equations in math. To the common nonlinear equations, the solution algorithm is mature in theory[4]. But the cooperation equations from the aeroengine model are so complex that the general methods such as Newton-Rphason method do not work usually. The equations' characters can be described as strong nonlinear, having no explicit expression, high division, gradient information getting hard, and so on, since the working process of every component and the whole engine is highly complex aerothermodynamic process. It will be difficult to be convergent, easy to be interrupted during iteration, and highly depending on the iteration initial value when using the traditional N-R algorithm[5].

The cooperation equations can be conversed to nonlinear least-squares problem by least-squares criterion. Then the aeroengine model solution is relevant with optimization problem closely, so the nonlinear model can be solved using optimization algorithm, which can avoid the difficulties when using common numerical in some degree. There are some researches about solving aerogine with modern optimization algorithms based on searching in random, such as Genetic algorithm (GA), Particle Swarm Optimization Algorithm (PSO), and so on. But the problem of huge calculation quantity can't be resolved throughout, since both the algorithms and the model is very complex[6]. So an improved optimization algorithm based on iteration calculation is studied to solve the nonlinear model.

\section{NONLINEAR MODEL AND THE PROBLEM CONVERSION}

\section{A. Nonlinea Model of Aero Engine}

The aeroengine component level model is established by variable specific heat method, which is mature relatively in modeling process and theory. Taken a given turbofan engine as an example, which is a low-bypass ratio, twin-spool, afterburning turbofan engine. The independent variables are chosen based on control rule, which is design as keeping the low-pressure rotor speed constant by control the fuel flow. Then the unknown operation variables $\boldsymbol{x}_{e}$ is conformed as:

$$
\boldsymbol{x}_{e}=\left[\beta_{c L}, n_{H}, \beta_{c H}, T_{4}^{*}, d h_{T H}^{*}, d h_{T L}^{*}\right]
$$

Where $\beta_{c L}$ and $\beta_{c H}$ stand for working position parameter of low and high-pressure compressor, $\bar{n}_{2 c o r}$ stands for conversion speed of high rotors, $T_{4}^{*}$ stand for total temperature before high turbine, $d h_{T H}^{*}$ and $d h_{T L}^{*}$ stand for enthalpy descending of highpressure and low-pressure turbine

Each component's aerothermodynamic model is established according to the sequence of airflow flowing through gas path from inlet to nozzle.

According to the requirement of working cooperation, the balance equations are established as follows using residual form[4].

Continuity equation for flow at outlet of low-pressure turbine:

$$
e r r_{1}=\left(M_{44}-M_{44}^{\prime}\right) / M_{44}
$$

Continuity equation for flow at outlet of high-pressure turbine:

$$
e r r_{2}=\left(M_{40}-M_{40}^{\prime}\right) / M_{40}
$$

Balance equation for power of low-pressure spool: 


$$
\operatorname{err}_{3}=\left(N_{T L}-N_{c L}\right) / N_{T L}
$$

Balance equation for power of high-pressure spool:

$$
e r r_{4}=\left(N_{T H}-N_{c H}\right) / N_{T H}
$$

Balance equation for static pressure at mixer inlet:

$$
\operatorname{err}_{5}=\left(P_{5}-P_{15}\right) / P
$$

Continuity equation for flow at inlet of nozzle:

$$
\operatorname{err}_{6}=\left(M_{8}-M_{7}\right) / M_{8}
$$

Where $M$ stands for flow value, $P$ stands for static pressure value, $N$ stands for power value. The subscript $C$ stands for compressor, $T$ stands for turbine, $L$ stands for low-pressure spool, $H$ stands for high-pressure spool, and the other figure subscripts stand for different boundary identification of the engine.

\section{B. Conversion of the Problem}

The problem of cooperation working equations solution can be conversed to a single object optimization problem, so the object function should be got firstly. The equations (1)-(6) can be written as follows:

$$
\boldsymbol{e r r}(\boldsymbol{x})=\boldsymbol{e r r}\left(x_{1}, x_{2}, \cdots, x_{6}\right)=\left\{\begin{array}{c}
\operatorname{err}_{1}\left(x_{1}, x_{2}, \cdots, x_{6}\right) \\
\operatorname{err}_{2}\left(x_{1}, x_{2}, \cdots, x_{6}\right) \\
\vdots \\
\operatorname{err}_{6}\left(x_{1}, x_{2}, \cdots, x_{6}\right)
\end{array}\right.
$$

In terms of the least square principle, the formula (7) are equal to the problem of (8):

$$
\min f(x)=\operatorname{err}(\boldsymbol{x})^{T} \operatorname{err}(\boldsymbol{x})=\sum_{i=1}^{6} \operatorname{err}_{i}^{2}\left(x_{1}, x_{2}, \cdots, x_{10}\right)
$$

Then the equations solution is conversed to a single object optimization problem, and the object function is shown as (8)

\section{THE HYBRID ITERATION OPTIMIZATION ALGORITHM DESIGN}

\section{A. The Difficulties of the Usual Optimization Algorithm to Complex Problem}

The difficulties of the usual optimization algorithm to complex problem

The basic algorithm for nonlinear optimization problem is the Newton method, by which it is difficulty to solve the complex problems. The basic Newton method can be improved according to the different natures of different object functions, such as Gauss-Newton (G-N) method, LevenbergMarquardt (L-M) method based on trust-region, then the difficulties of usual algorithm can be improved in some degree. The equal least-square question is as difficult as the aeroengine model, whose characters conclude high dimension, strong nonlinear, implicit expression, and so on. This leastsquare problem is too complex to be solved by using variable step factor G-N or L-M directly, and the QR decomposition can't completely resolve the Jacobian matrix condition number(which will get to $1 \mathrm{e} 9$ 1e10 magnitude) getting worse also. Further more, the singular value decomposition (SVD) method is reliable to improve the divergence problem caused for matrix condition worsening, but the calculation amount is too huge to fit for the complex engine model solution.

\section{B. L-M and $G-N$ Algorithm}

According to the nonlinear optimization , the Newton method for the common nonlinear question described as $\min f(\boldsymbol{x})$ is:

$$
x_{k+1}=x_{k}-\left[\nabla f^{2}\left(x_{k}\right)\right]^{-1} \nabla f\left(x_{k}\right)
$$

Where $\nabla f\left(\boldsymbol{x}_{k}\right)$ is gradient, $\nabla f^{2}\left(\boldsymbol{x}_{k}\right)$ is the Hessian matrix.

The basic Newton method is a method in theory, to most actual questions, because of the difficulties such as the Hessian matrix calculation, convergence. The Gauss-Newton with variable step-size factor and Levenberg-Marqurdt is the two common improved methods.

(1) Gauss-Newton with variable step-size factor

To the formula (9), the basic Newton method, the Hessian Matrix can be approximated by $\boldsymbol{J}\left(\boldsymbol{x}_{k}\right)^{T} \boldsymbol{J}\left(\boldsymbol{x}_{k}\right)$, then the Gauss-Newton algorithm with variable step-size factor $\alpha_{k}$ iteration can be gotten:

$$
\boldsymbol{x}_{k+1}=\boldsymbol{x}_{k}-\alpha_{k}\left[\boldsymbol{J}\left(\boldsymbol{x}_{k}\right)^{T} \boldsymbol{J}\left(\boldsymbol{x}_{k}\right)\right]^{-1} \boldsymbol{J}\left(\boldsymbol{x}_{k}\right)^{T} \boldsymbol{E}\left(\boldsymbol{x}_{k}\right)
$$

The introduction of $\alpha_{k}$ is to ensure the descending direction of searching, which is described as $f\left(x_{k+1}\right) \leq f\left(x_{k}\right)$. So the solution of $\alpha_{k}$ is a problem of one-dimension optimization problem as (10), which is to minimize the object function of $k$ step iteration.

$$
\min _{\alpha} f\left(\boldsymbol{x}_{k}-\alpha_{k}\left[\boldsymbol{J}\left(\boldsymbol{x}_{k}\right)^{T} \boldsymbol{J}\left(\boldsymbol{x}_{k}\right)\right]^{-1} \boldsymbol{J}\left(\boldsymbol{x}_{k}\right)^{T} \boldsymbol{E}\left(\boldsymbol{x}_{k}\right)\right)
$$

The one-dimension optimization problem is not difficulty to solve, but the calculation quantity will increase, though the process is also a iteration process.

(2) Levenberg-Marqurdt algoritm

Levenberg-Marqurdt is an improved algorithm based on trust-region.In terms of the optimization theory, the nonlinear 
least square problem is different from the common problem, which can be solved using linear least square solution algorithm by conversion. The simplified linear iteration is:

$$
\bar{f}(x)=f\left(x_{k}\right)+J\left(x_{k}\right)\left(x-x_{k}\right)
$$

But the initial $f(x)$ is nonlinear, so the linearization model can not be insured exact in the whole definition domain, so the trust region model is introduced. The idea is the linearization model is exact in the given region, and the problem is conversed to a least square problem with constraint. In the trust region model, the neighborhood is defined by limiting the up boundary. The neighborhood of $\boldsymbol{x}_{k}$ is (13), when the up boundary is $h_{k}$.

$$
\Omega_{k}=\left\{\boldsymbol{x} \mid\left\|\boldsymbol{x}-\boldsymbol{x}_{k}\right\| \leq h_{k}\right\}
$$

Then the trust region model of G-N is described as (14).

$$
\begin{cases}\min & \frac{1}{2} \overline{\boldsymbol{E}}(\boldsymbol{x})^{T} \overline{\boldsymbol{E}}(\boldsymbol{x}) \\ \text { s.t. } & \left(\boldsymbol{x}-\boldsymbol{x}_{k}\right)^{T}\left(\boldsymbol{x}-\boldsymbol{x}_{k}\right) \leq h_{k}{ }^{2}\end{cases}
$$

Introduce step length $\delta \boldsymbol{x}_{k}$, the model is changed to (15), where the norm can be defined by many format. The Levenberg-Marqurdt method is gotten, when the 2-norm is applied[7,8].

\section{Design of Improved Algorithm}

To the complex optimization problems, the improved G-N algorithm applying QR decomposition can improve the matrix condition number worsening, but sometimes it will also be unsatisfied when the object function is too complex. The improved L-M algorithm with QR decomposition, a kind of trust-region methods, will get robust sometimes, but some procedure parameters are difficult to compute. So an improved hybrid algorithm is proposed, that combine G-N introduced variable step factor with L-M to get both advantages, and the QR composition is also applied in the process of iterations, by which the aeroengine nonlinear model is solved. There are so many books and papers introducing the basic theory of the classic optimization algorithms in detail, so we will not repeat those again.

According to the complexity of the problem, an improved algorithm is design. The basic flow of the improved algorithm is shown as figure 1 . When the solution process is starting, the first step begins from G-N method in order to save calculate resource, whose iteration procedure is simple relatively. During the iteration calculation, the step value is checked, the L-M method will begin when the step is smaller than the given threshold. Because if the step value is too small, the descend speed of optimization becomes too slow to improve the solution obviously. Considered that the step becoming small may be caused of the Jacobian matrix condition worsening, and the L-M method is more excellent to solve the problem.So the optimization iteration is switched to L-M method. That is why the hybrid algorithm is proposed as above. The variables of the engine model is described as vector, then the iteration step is also a vector, so the threshold value to control the switching of two algorithm is chosen as 2-norm.

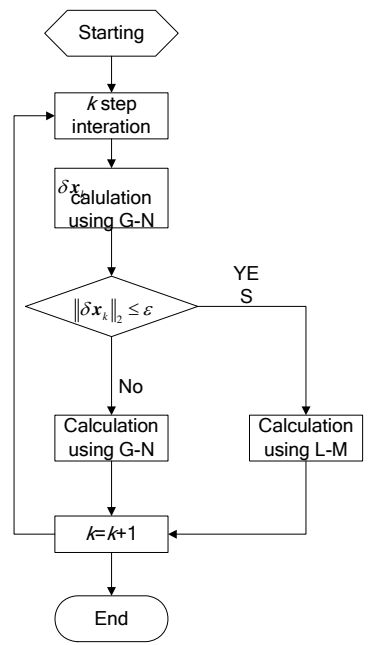

FIGURE I. FLOW OF IMPROVED HYBRID ALGORITHM

\section{SiMULATION EXAMPLE AND ANALYSIS}

Taken a given turbofan engine as example. The iteration steps can't be too many in order to ensure the calculation speed, so the simulation steps are limited to 20 . The aeroengine model is solved applying the new improved hybrid optimization method and G-N method, and the initial value is given the same in order to compare the algorithms performance. The iteration process curve is shown as figure 2 and figure 3 . The results show that the computation precision of improved algorithm is higher than the traditional G-N method, which uses the QR decomposition also, and the iteration precision can reach to $1 \mathrm{e}-5$.

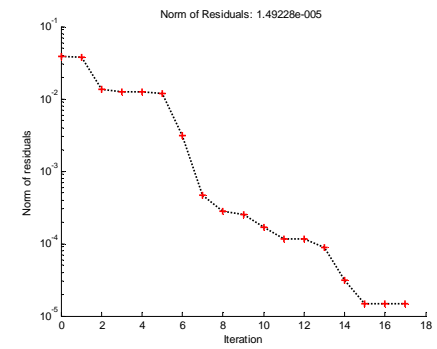

FIGURE II. RESIDUALS CURVE OF IMPROVED ALGORITHM ITERATION 


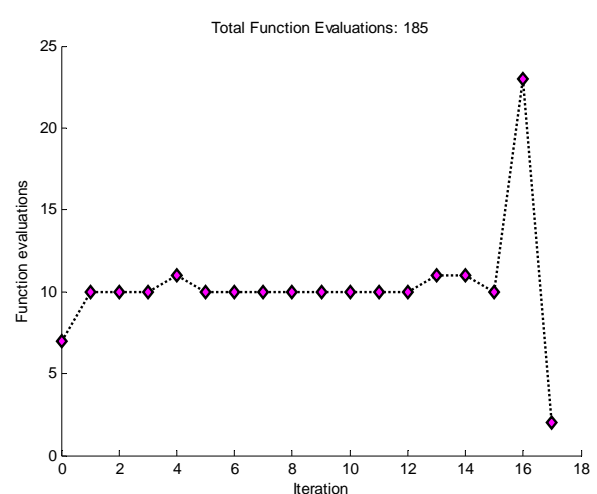

FIGURE III. TOTAL FUNCTION EVALUATIONS OF IMPROVED ALGORITHM

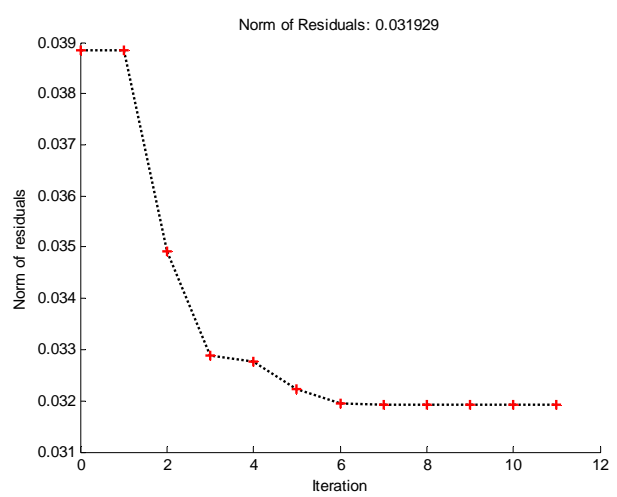

FIGURE IV. RESIDUALS CURVE OF G-N ITERATION

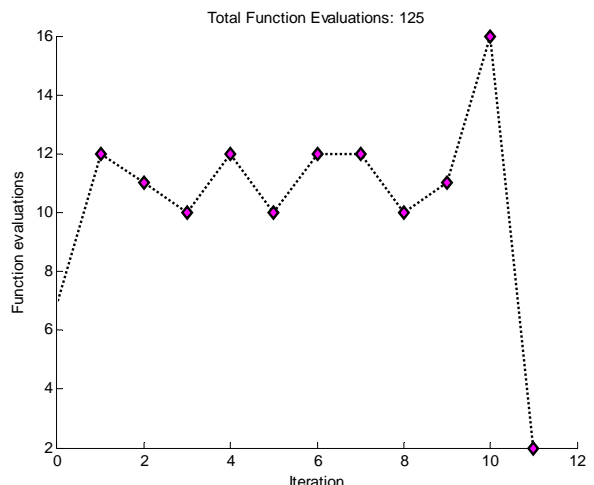

FIGURE V. TOTAL FUNCTION EVALUATIONS OF G-N

\section{CONCLUSIONS}

The traditional optimization method based on iteration has their own limitation, and every classic optimization algorithm can't be improved to fit for all of the optimization problems, either can get rid of the dependence on the initial guess values. But in many conditions the calculation speed is very important, so the traditional iteration optimization is necessary to be used. The mathematical character of turbofan engine model is so complex, that the equal nonlinear optimization question is also complex. The object function has many local maximum points, minimum points, and saddle points, it is difficult to solve use traditional iteration optimization method. According to the simulation result, though the algorithm is improved, it is also very difficult to get the whole optimization solutions. The purpose of studying algorithm improvement is to get higher probability and faster speed of convergence, and to decrease the solution's dependence on the initial guess value. It is clear that if the initial value is given in random, the whole optimization solution can't be gotten. But to the specific question, the initial values are always given according to the background, or the optimization solution can’t be acquired, however the algorithm is improved.

\section{REFERENCE}

[1] Behabhani A, Adibhatla S, Rauche C. Integrated Model-Based Controls and PHM for Improving Turbine Engine Performance, Reliability, and Cost. 45th AIAA/ASME/SAE/ASEE Joint Propulsion Conference \& Exhibit,Denver, Colorado: 2009.

[2] Litt Jonathan S., Simon Donald L. A Survey of Intelligent Control and Health Management Technologies for Aircraft Propulsion System. Journal of Aerospace Computiong, Information, and Communicaton. 2004, 1: 543-563.

[3] Lytle J. K., Follen G. J., G Naiman C. Numerical Propulsion System Simulation (NPSS) 1999 Industry Review. NASA TM- 2000 - 209795, 2000 .

[4] Sellers J. F., Carl J. Danielle. DYNGEN-A Program for Calculating Steady-state and Transient Performance of Turbojet and Turbofan Engine. NASA TN-D-7901, 1973.

[5] Chen Y Ch, Xu S Y, Yang Y K, etal. Research on the method to solve convergence problem in aeroturbo-engine performance computation. Journal of Aerospace Power, Vol 23, 2242-2248,2008

[6] L. Song-lin and S. Jian-gu, "Application of Genetic Algorithm to Solving Nonlinear Model of Aeroengines," CHINESE JOURNAL OF AERONAUTICS , vol. 16, 2003.

[7] Wei Z, Li G, Qi L. New quasi-Newton methods for unconstrained optimization problems. Applied Math-ematics and Computation, 175: 1156-1188, 2006.

[8] Yuan Y X, Sun W Y.Optimization Theory and Methods.Beijing: Science Press, 1997. 